\title{
Quantum Nonlocality of N-qubit W states
}

\author{
Chunfeng Wu, ${ }^{1}$ Jing-Ling Chen, ${ }^{2}$ L. C. Kwek, ${ }^{1,3}$ and C. H. Oh ${ }^{1, \text { F }}$ \\ ${ }^{1}$ Department of Physics, National University of Singapore, 2 Science Drive 3, Singapore 117542 \\ ${ }^{2}$ Theoretical Physics Division, Nankai Institute of Mathematics, \\ Nankai University, Tianjin 300071, P. R. China \\ ${ }^{3}$ Nanyang Technological University, National Institute of Education, 1, Nanyang Walk, Singapore 637616
}

\begin{abstract}
An experimental setup for testing quantum nonlocality of $\mathrm{N}$ qubits is proposed. This method is a generalization of the optical setup proposed by Banaszek and Wódkiewicz [1]. The quantum nonlocality of $\mathrm{N}$ qubits can be obtained through its violation of $\mathrm{N}$-qubit Bell inequalities. The correlation function measured in the experiment is described by the Wigner function. The effect of inefficient detector is also considered.
\end{abstract}

PACS numbers: 03.65.Ud, 03.67.-a, 42.50.Dv

\section{INTRODUCTION}

Experimental verification on the conflict between quantum mechanics and local realism for two particles have been well demonstrated in several experiments 2, 3]. For a system with more than two particles, for example three particles, experimental verification of the violation of local realism is generally more difficult. Recently, conflicts between quantum mechanics and local realism for three qubits and four qubits have also been verified [4, 5]. For $\mathrm{N}$ qubits, experimental observations for violation of Bell inequalities are still lacking.

In Ref. 1, the authors proposed an optical setup for testing quantum nonlocality in phase space for two qubits. The setup essentially demonstrate quantum nonlocality based on phase space measurement of the Wigner function using photon counting. The source used in Ref. 1] is a single photon incident on a 50:50 beam splitter (see Figure 11). The generated state is $|\psi(2)\rangle=$ $\frac{1}{\sqrt{2}}(|10\rangle \pm|01\rangle)$ written in terms of the exit ports $a_{1}$ and $a_{2}$. For example, $|10\rangle$ means one photon exits at port $a_{1}$ and no photon exits at port $a_{2}$. \pm can be realized by one phase shifter to adjust the relative phase and the relative phase can be set to zero without loss of generality. As pointed in Ref. [1], the measuring apparatus consists of a beam splitter and photon counting detector. The power transmission of the beam splitter is $\mathrm{T}$. The incidence of an excited coherent state $|\gamma\rangle$ on the second input port of the beam splitter results in coherent displacement. Namely, the action of the beam splitter is described by $\hat{D}(\sqrt{1-T} \gamma)$ in the limit of $T \rightarrow 1$ and $\gamma \rightarrow \infty$. The realistic measurement proposed in Ref. [1] is a test that the detectors are capable of resolving the number of absorbed photons and $+1 /-1$ are assigned correspondingly to events in which an even/odd number of photons is registered. In short, the correlation function measured is the Wigner function. In the experiment, quantum nonlocality of two qubits is revealed through

\footnotetext{
*Electronic address: phyohch@nus.edu.sg
}

violation of the $\mathrm{CHSH}$ inequality [6]. In this paper, we generalize the method to a $N$-particle system.

For $N$ qubits, there have been umpteen Bell inequalities that have been proposed 7, 8, 9, 10, 11] to test quantum nonlocality. The more general inequalities are the ones given in [10, 11]. These inequalities (ŻB inequalities) impose sufficient and necessary conditions for the existence of local and realistic model. The ŻB inequalities take the form

$$
\begin{aligned}
& \mid \sum_{s_{1}, \ldots s_{N}= \pm 1} S\left(s_{1}, \ldots s_{N}\right) \times \\
& \quad \sum_{k_{1}, \ldots k_{N}=1,2} s_{1}^{k_{1}-1} \ldots s_{n}^{k_{N}-1} E\left(k_{1}, \ldots k_{N}\right) \mid \leq 2^{N},
\end{aligned}
$$

where $E\left(k_{1}, \ldots k_{N}\right)$ is correlation function measured in a dichotomic-observable experiment. Specific choices give nontrivial inequalities. For example, for $S\left(s_{1}, \ldots, s_{N}\right)=$ $\sqrt{2}\left(\cos \left[-\frac{\pi}{4}+\left(s_{1}+\ldots+s_{N}-N\right) \frac{\pi}{4}\right]\right)$ one recovers the MABK inequalities $[7,8,9]$. In the article, we propose a general experimental setup for testing quantum nonlocality of $N$ qubits by using the ŻB inequalities. In section【 we generalize the two-qubit experimental scheme to $N$ qubits. We provide the general form of the correlation functions measured in the scheme. In section [III we consider the cases for $N=3,4$ as examples to show that the experimental observation of quantum nonlocality can be linked to the violation of 3-qubit and 4-qubit Bell inequalities. For three qubits, the ŻB inequality is not violated by all pure entangled states 12, 13. In Refs. 14, 15], we constructed Bell inequalities which are violated by any pure entangled state of three qubits. Both 3-qubit ŻB inequality and the Bell inequalities given in Refs. 14, 15 will be used to test nonlocality in the proposed experiment. Effect of detector inefficiency is also considered in the section.

\section{PROPOSED EXPERIMENT FOR TESTING QUANTUM NONLOCALITY}

We first consider the source for generating an entangled state of $N$ qubits for the optical setup. For two qubits, 


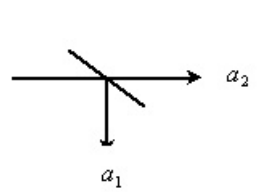

(1)

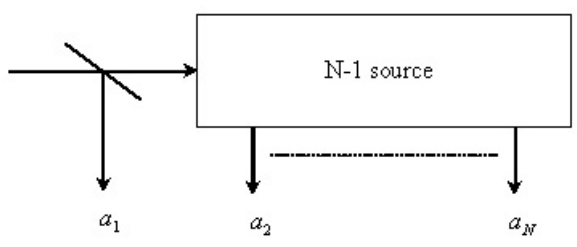

(3)

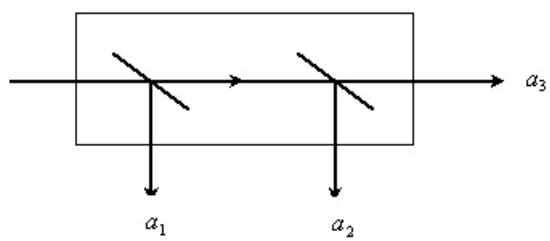

(2)
FIG. 1: The sources proposed to generate N-qubit entangled states. In (1), the beam splitter is a 50:50 one which generates a two-qubit $\mathrm{W}$ state (a Bell state). In (2), the first beam splitter has a reflectivity $R=\frac{1}{3}$ and the second beam splitter is a 50:50 one. These two beam splitters generate a threequbit $\mathrm{W}$ state. We have used a black box to represent the source. In (3), the beam splitter has a reflectivity $R=\frac{1}{N}$. The box represents a source which generates a (N-1)-qubit W state. The action of the beam splitter and the (N-1) source is to generate a $\mathrm{N}$-qubit $\mathrm{W}$ state.

the source is a 50:50 beam splitter. The output state of the source is $|\psi(2)\rangle=\frac{1}{\sqrt{2}}(|10\rangle+|01\rangle)$. For three qubits, we look for a source that consists of a beam splitter with reflectivity $R=\frac{1}{3}$ followed by a 50:50 beam splitter. The output state is $|\psi(3)\rangle=\frac{1}{\sqrt{3}}(|100\rangle+|010\rangle+|001\rangle)$. Such a source is shown in Figure 1(2). In the Figure 1 (2), we use a black box to represent an effective three-qubit source. For a four-qubit source, one can build on the three-qubit source with an additional beam splitter. The beam splitter in this case has a reflectivity $R=\frac{1}{4}$. Generalizing, an $N$-qubit source for arbitrary $N$ qubit is realized by a beam splitter with reflectivity $R=\frac{1}{N}$ followed by a $(N-1)$-qubit source. The overall action of $(N-1)$ qubit source is that a single photon entering the box will have equal chances of exiting at any of the $(N-1)$ ports. The probability is $\frac{1}{N-1}$. The beam splitter has a transmittance $T=\frac{N-1}{N}$. So in Figure 1 (3), the probability of a single photon exiting from each port $\left(a_{2}, \ldots a_{N}\right)$ of the box is $\frac{1}{N-1} \frac{N-1}{N}=\frac{1}{N}$. The probability of a single photon exiting from the reflected port $a_{1}$ of the beam splitter is also $\frac{1}{N}$ since its reflectivity $R=\frac{1}{N}$. Therefore the effective source generates an $N$-qubit $\mathrm{W}$ state $|\psi(N)\rangle=\frac{1}{\sqrt{N}}(|10 \ldots 0\rangle+|01 \ldots 0\rangle+\ldots+|0 \ldots 01\rangle)$.

The experimental setup is schematically shown in Figure2 A single photon enters the source and exits with an equal chance at each outgoing mode $a_{i}$. The measuring

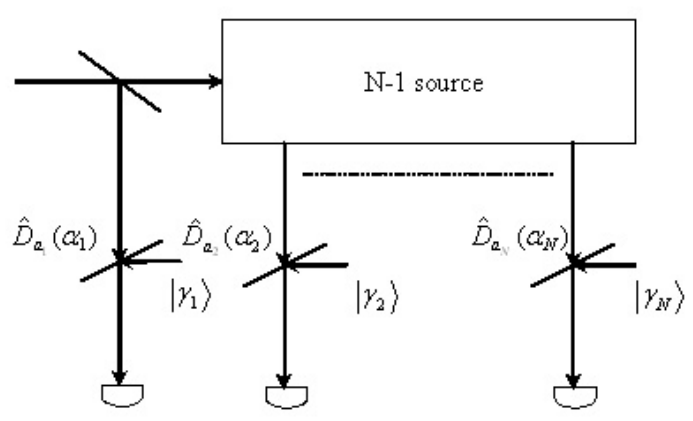

FIG. 2: The optical setup for testing nonlocality of $\mathrm{N}$ qubits. The beam splitter in the top line, which has a reflectivity $R=1 / N$, and the $(\mathrm{N}-1)$ source generate a N-qubit entangled state. Coherent displacements $\hat{D}_{a_{i}}\left(\alpha_{i}\right)$ are realized by strong coherent states $\left|\gamma_{i}\right\rangle\left(\gamma_{i} \rightarrow \infty\right)$ injected into beam splitters which have transmittance close to $1\left(T_{i} \rightarrow 1\right)$. N detectors are put in the lowest line.

devices are photon detectors preceded by beam splitters which have transmittance $T_{i} \rightarrow 1$. Strong coherent states $\left|\gamma_{i}\right\rangle$ are injected into the beam splitters. As a result, the beam splitters give coherent displacements $\hat{D}_{a_{i}}\left(\alpha_{i}\right)$ with $\alpha_{i}=\sqrt{1-T_{i}} \gamma_{i}$ on the input modes when $\gamma_{i} \rightarrow \infty[1]$. By assigning $+1 /-1$ to the events that an even/odd number of photons has been registered, the correlation function measured in the experiment is given by

$$
\begin{aligned}
& \Pi_{a_{1} a_{2} \ldots a_{N}}\left(\alpha_{1}, \alpha_{2}, \ldots, \alpha_{N}\right)= \\
& \left\langle\psi(N)\left|\hat{\Pi}_{a_{1}}\left(\alpha_{1}\right) \otimes \hat{\Pi}_{a_{2}}\left(\alpha_{2}\right) \otimes \ldots \otimes \hat{\Pi}_{a_{N}}\left(\alpha_{N}\right)\right| \psi(N)\right\rangle,
\end{aligned}
$$

where $\alpha_{i}$ is coherent displacement for the mode $a_{i}$. $\hat{\Pi}_{a_{i}}\left(\alpha_{i}\right)$ is an operator defined as [1]

$$
\begin{aligned}
\hat{\Pi}_{a_{i}}\left(\alpha_{i}\right)= & \hat{D}_{a_{i}}\left(\alpha_{i}\right) \sum_{k=0}^{\infty}|2 k\rangle\langle 2 k| \hat{D}_{a_{i}}^{\dagger}\left(\alpha_{i}\right) \\
& -\hat{D}_{a_{i}}\left(\alpha_{i}\right) \sum_{k=0}^{\infty}|2 k+1\rangle\langle 2 k+1| \hat{D}_{a_{i}}^{\dagger}\left(\alpha_{i}\right) .
\end{aligned}
$$

The operator $\hat{\Pi}_{a_{i}}\left(\alpha_{i}\right)$ is also of the form [1]

$$
\hat{\Pi}_{a_{i}}\left(\alpha_{i}\right)=\hat{D}_{a_{i}}\left(\alpha_{i}\right)(-1)^{\hat{n}_{a_{i}}} \hat{D}_{a_{i}}^{\dagger}\left(\alpha_{i}\right),
$$

which is the displaced parity operator. Because that

$$
\begin{aligned}
\hat{D}_{a_{i}}^{\dagger}\left(\alpha_{i}\right)|0\rangle= & e^{-\frac{1}{2}\left|\alpha_{i}\right|^{2}} \sum_{m}\left(-\alpha_{i}\right)^{m} \frac{1}{\sqrt{m !}}|m\rangle \\
\hat{D}_{a_{i}}^{\dagger}\left(\alpha_{i}\right)|1\rangle= & e^{-\frac{1}{2}\left|\alpha_{i}\right|^{2}}\left\{\sum_{m}\left(-\alpha_{i}\right)^{m} \alpha_{i}^{*} \frac{1}{\sqrt{m !}}|m\rangle\right. \\
& \left.+\sum_{m}\left(-\alpha_{i}\right)^{m} \frac{\sqrt{m+1}}{\sqrt{m !}}|m+1\rangle\right\}
\end{aligned}
$$


for the state $|\Psi(N)\rangle$, we have

$$
\begin{aligned}
& \Pi_{a_{1} a_{2} \ldots a_{N}}\left(\alpha_{1}, \alpha_{2}, \ldots, \alpha_{N}\right)= \\
& \frac{1}{N}\left(4\left|\sum_{i=1}^{N} \alpha_{i}\right|^{2}-N\right) e^{-2 \sum_{i=1}^{N}\left|\alpha_{i}\right|^{2}} .
\end{aligned}
$$

For $N$ qubits, by measuring correlation functions and using $\dot{Z} B$ inequalities, one can hopefully show violation of $N$-qubit Bell inequality. In the next section, we investigate quantum nonlocality of 3 and 4 qubits.

\section{EXAMPLES}

In this section, we generalize the scheme to 3 qubits first. For three qubits, the source of nonclassical radiation is a single photon incident on a beam splitter with transmittance $T=2 / 3$ and reflectivity $R=1 / 3$ followed by a 50:50 beam splitter, which generates a three-qubit $W$ state. The measuring devices are photon counters with beam splitters placed in front of them. The beam splitters have the transmission coefficient close to one with strong coherent beams injected into the auxiliary ports. In this limit, the beam splitters effectively perform coherent displacements $\hat{D}_{a_{1}}\left(\alpha_{1}\right), \hat{D}_{a_{2}}\left(\alpha_{2}\right)$ and $\hat{D}_{a_{3}}\left(\alpha_{3}\right)$ on the three ports (modes) of the input field. The correlation function measured is

$$
\begin{aligned}
& \Pi_{a_{1} a_{2} a_{3}}\left(\alpha_{1}, \alpha_{2}, \alpha_{3}\right) \\
& =\left\langle\psi(3)\left|\hat{\Pi}_{a_{1}}\left(\alpha_{1}\right) \otimes \hat{\Pi}_{a_{2}}\left(\alpha_{2}\right) \otimes \hat{\Pi}_{a_{3}}\left(\alpha_{3}\right)\right| \psi(3)\right\rangle \\
& =\frac{1}{3} e^{-2\left(\left|\alpha_{1}\right|^{2}+\left|\alpha_{2}\right|^{2}+\left|\alpha_{3}\right|^{2}\right)}\left(4\left|\alpha_{1}+\alpha_{2}+\alpha_{3}\right|^{2}-3\right) .
\end{aligned}
$$

We next construct a Bell quantity from the 3-qubit $\dot{Z} B$ inequality:

$$
\begin{aligned}
\mathcal{B}_{3 \mathrm{qubits}} & =\Pi_{a_{1} a_{2} a_{3}}\left(\alpha_{1}^{1}, \alpha_{2}^{1}, \alpha_{3}^{2}\right)+\Pi_{a_{1} a_{2} a_{3}}\left(\alpha_{1}^{1}, \alpha_{2}^{2}, \alpha_{3}^{1}\right) \\
& +\Pi_{a_{1} a_{2} a_{3}}\left(\alpha_{1}^{2}, \alpha_{2}^{1}, \alpha_{3}^{1}\right)-\Pi_{a_{1} a_{2} a_{3}}\left(\alpha_{1}^{2}, \alpha_{2}^{2}, \alpha_{3}^{2}\right)
\end{aligned}
$$

where $\alpha_{j}^{i}$ with $i=1,2$ and $j=1,2,3$ are the two experimental settings of the coherent displacements for ports $a_{1}, a_{2}$ and $a_{3}$ respectively. For a local realistic theory, $\mathcal{B}_{3 q u b i t s} \leq 2$. Unfortunately 3 -qubit $\dot{\mathrm{ZB}}$ inequality does not reveal quantum nonlocality since a numerical calculation gives $\mathcal{B}_{3 q u b i t s} \leq 2$. A possible reason is that the degree of quantum nonlocality depends not only on the given entangled state but also on the Bell operator [16]. Hence the result means that 3 -qubit $\dot{Z} B$ inequality may not reveal quantum nonlocality for displacement measurements on the particles.

Recently new Bell inequalities for three qubits have been proposed in Refs. 14, 15]. The Bell inequalities are violated for any pure entangled state. We next show that quantum nonlocality of three qubits can be exhibited in the proposed experimental scheme using the correlationform inequality given in [15].
Unlike the 3-qubit $\dot{Z} B$ inequality where there are only three-particle correlation functions, the recent Bell inequality for three qubits contains two-particle correlation functions and one-particle correlation functions [15]. The two-particle correlation functions and one-particle correlation functions are given by

$$
\begin{aligned}
& \Pi_{a_{1} a_{2}}\left(\alpha_{1}, \alpha_{2}\right)=\left\langle\psi(3)\left|\hat{\Pi}_{a_{1}}\left(\alpha_{1}\right) \otimes \hat{\Pi}_{a_{2}}\left(\alpha_{2}\right) \otimes \mathbf{1}\right| \psi(3)\right\rangle, \\
& \Pi_{a_{1} a_{3}}\left(\alpha_{1}, \alpha_{3}\right)=\left\langle\psi(3)\left|\hat{\Pi}_{a_{1}}\left(\alpha_{1}\right) \otimes \mathbf{1} \otimes \hat{\Pi}_{a_{3}}\left(\alpha_{3}\right)\right| \psi(3)\right\rangle, \\
& \Pi_{a_{2} a_{3}}\left(\alpha_{2}, \alpha_{3}\right)=\left\langle\psi(3)\left|\mathbf{1} \otimes \hat{\Pi}_{a_{2}}\left(\alpha_{2}\right) \otimes \hat{\Pi}_{a_{3}}\left(\alpha_{3}\right)\right| \psi(3)\right\rangle, \\
& \Pi_{a_{1}}\left(\alpha_{1}\right)=\left\langle\psi(3)\left|\hat{\Pi}_{a_{1}}\left(\alpha_{1}\right) \otimes \mathbf{1} \otimes \mathbf{1}\right| \psi(3)\right\rangle, \\
& \Pi_{a_{2}}\left(\alpha_{2}\right)=\left\langle\psi(3)\left|\mathbf{1} \otimes \hat{\Pi}_{a_{2}}\left(\alpha_{2}\right) \otimes \mathbf{1}\right| \psi(3)\right\rangle, \\
& \Pi_{a_{3}}\left(\alpha_{3}\right)=\left\langle\psi(3)\left|\mathbf{1} \otimes \mathbf{1} \otimes \hat{\Pi}_{a_{3}}\left(\alpha_{3}\right)\right| \psi(3)\right\rangle .
\end{aligned}
$$

The two-particle correlation functions are measured when one of three observers does not perform any measurement on his detector. The one-particle correlation functions are measured when two of three observers do not perform any measurement on their detectors. By similar calculations to the one for three-particle correlation function, we have

$$
\begin{aligned}
& \Pi_{a_{i} a_{j}}\left(\alpha_{i}, \alpha_{j}\right)=\frac{1}{3} e^{-2\left(\left|\alpha_{i}\right|^{2}+\left|\alpha_{j}\right|^{2}\right)}\left(4\left|\alpha_{i}+\alpha_{j}\right|^{2}-1\right) \\
& \Pi_{a_{i}}\left(\alpha_{i}\right)=\frac{1}{3} e^{-2\left(\left|\alpha_{i}\right|^{2}\right)}\left(4\left|\alpha_{i}\right|^{2}+1\right)
\end{aligned}
$$

where $i, j=1,2,3$. Using a correlation form of the inequalities for three qubits given in Ref. 15], we construct a new Bell quantity for three qubits

$$
\begin{aligned}
\mathcal{B}_{3 \mathrm{qubits}}^{\prime} & =-\Pi_{a_{1} a_{2} a_{3}}\left(\alpha_{1}^{1}, \alpha_{2}^{1}, \alpha_{3}^{1}\right)+\Pi_{a_{1} a_{2} a_{3}}\left(\alpha_{1}^{1}, \alpha_{2}^{1}, \alpha_{3}^{2}\right) \\
& +\Pi_{a_{1} a_{2} a_{3}}\left(\alpha_{1}^{1}, \alpha_{2}^{2}, \alpha_{3}^{1}\right)+\Pi_{a_{1} a_{2} a_{3}}\left(\alpha_{1}^{2}, \alpha_{2}^{1}, \alpha_{3}^{1}\right) \\
& -\Pi_{a_{1} a_{2} a_{3}}\left(\alpha_{1}^{2}, \alpha_{2}^{2}, \alpha_{3}^{2}\right)-\Pi_{a_{1} a_{2}}\left(\alpha_{1}^{1}, \alpha_{2}^{2}\right) \\
& -\Pi_{a_{1} a_{2}}\left(\alpha_{1}^{2}, \alpha_{2}^{1}\right)-\Pi_{a_{1} a_{2}}\left(\alpha_{1}^{2}, \alpha_{2}^{2}\right) \\
& -\Pi_{a_{1} a_{3}}\left(\alpha_{1}^{1}, \alpha_{3}^{2}\right)-\Pi_{a_{1} a_{3}}\left(\alpha_{1}^{2}, \alpha_{3}^{1}\right) \\
& -\Pi_{a_{1} a_{3}}\left(\alpha_{1}^{2}, \alpha_{3}^{2}\right)-\Pi_{a_{2} a_{3}}\left(\alpha_{2}^{1}, \alpha_{3}^{2}\right) \\
& -\Pi_{a_{2} a_{3}}\left(\alpha_{2}^{2}, \alpha_{3}^{1}\right)-\Pi_{a_{2} a_{3}}\left(\alpha_{2}^{2}, \alpha_{3}^{2}\right) \\
& +\Pi_{a_{1}}\left(\alpha_{1}^{1}\right)+\Pi_{a_{2}}\left(\alpha_{2}^{1}\right)+\Pi_{a_{3}}\left(\alpha_{3}^{1}\right) .
\end{aligned}
$$

Local realism theories impose the upper bound value of 3 for the Bell quantity $\mathcal{B}_{3 \text { qubits }}^{\prime}$ By taking the coherent displacements as $\alpha_{1}^{1}=\alpha_{2}^{1}=\alpha_{3}^{1}=0.471669, \alpha_{1}^{2}=\alpha_{2}^{2}=$ $\alpha_{3}^{2}=-0.0205849, \mathcal{B}_{3 \text { qubits }}^{\prime}=3.1605$ which is greater than 3 . Thus one can detect quantum nonlocality of a threequbit system in the proposed experiment.

For four qubits, the effective 4-qubit source generates a 4-qubit $\mathrm{W}$ state which takes the form

$$
|\psi(4)\rangle=\frac{1}{2}(|1000\rangle+|0100\rangle+|0010\rangle+|0001\rangle) .
$$

The correlation function measured is given by 


$$
\begin{aligned}
\Pi_{a_{1} a_{2} a_{3} a_{4}}\left(\alpha_{1}, \alpha_{2}, \alpha_{3}, \alpha_{4}\right)= & \left\langle\psi(4)\left|\hat{\Pi}_{a_{1}}\left(\alpha_{1}\right) \otimes \hat{\Pi}_{a_{2}}\left(\alpha_{2}\right) \otimes \hat{\Pi}_{a_{3}}\left(\alpha_{3}\right) \otimes \hat{\Pi}_{a_{4}}\left(\alpha_{4}\right)\right| \psi(4)\right\rangle \\
& =\frac{1}{4} e^{-2\left(\left|\alpha_{1}\right|^{2}+\left|\alpha_{2}\right|^{2}+\left|\alpha_{3}\right|^{2}+\left|\alpha_{4}\right|^{2}\right)}\left(4\left|\alpha_{1}+\alpha_{2}+\alpha_{3}+\alpha_{4}\right|^{2}-4\right) .
\end{aligned}
$$

Based on the 4-qubit ŻB inequality, the Bell quantity

$\mathcal{B}_{4 \text { qubits }}$ is constructed as

$$
\begin{aligned}
\mathcal{B}_{4 \mathrm{qubits}} & =\Pi_{a_{1} a_{2} a_{3} a_{4}}\left(\alpha_{1}^{1}, \alpha_{2}^{1}, \alpha_{3}^{1}, \alpha_{4}^{1}\right)-\Pi_{a_{1} a_{2} a_{3} a_{4}}\left(\alpha_{1}^{1}, \alpha_{2}^{1}, \alpha_{3}^{1}, \alpha_{4}^{2}\right)-\Pi_{a_{1} a_{2} a_{3} a_{4}}\left(\alpha_{1}^{1}, \alpha_{2}^{1}, \alpha_{3}^{2}, \alpha_{4}^{1}\right)-\Pi_{a_{1} a_{2} a_{3} a_{4}}\left(\alpha_{1}^{1}, \alpha_{2}^{1}, \alpha_{3}^{2}, \alpha_{4}^{2}\right) \\
& -\Pi_{a_{1} a_{2} a_{3} a_{4}}\left(\alpha_{1}^{1}, \alpha_{2}^{2}, \alpha_{3}^{1}, \alpha_{4}^{1}\right)-\Pi_{a_{1} a_{2} a_{3} a_{4}}\left(\alpha_{1}^{1}, \alpha_{2}^{2}, \alpha_{3}^{1}, \alpha_{4}^{2}\right)-\Pi_{a_{1} a_{2} a_{3} a_{4}}\left(\alpha_{1}^{1}, \alpha_{2}^{2}, \alpha_{3}^{2}, \alpha_{4}^{1}\right)+\Pi_{a_{1} a_{2} a_{3} a_{4}}\left(\alpha_{1}^{1}, \alpha_{2}^{2}, \alpha_{3}^{2}, \alpha_{4}^{2}\right) \\
& -\Pi_{a_{1} a_{2} a_{3} a_{4}}\left(\alpha_{1}^{2}, \alpha_{2}^{1}, \alpha_{3}^{1}, \alpha_{4}^{1}\right)-\Pi_{a_{1} a_{2} a_{3} a_{4}}\left(\alpha_{1}^{2}, \alpha_{2}^{1}, \alpha_{3}^{1}, \alpha_{4}^{2}\right)-\Pi_{a_{1} a_{2} a_{3} a_{4}}\left(\alpha_{1}^{2}, \alpha_{2}^{1}, \alpha_{3}^{2}, \alpha_{4}^{1}\right)+\Pi_{a_{1} a_{2} a_{3} a_{4}}\left(\alpha_{1}^{2}, \alpha_{2}^{1}, \alpha_{3}^{2}, \alpha_{4}^{2}\right) \\
& -\Pi_{a_{1} a_{2} a_{3} a_{4}}\left(\alpha_{1}^{2}, \alpha_{2}^{2}, \alpha_{3}^{1}, \alpha_{4}^{1}\right)+\Pi_{a_{1} a_{2} a_{3} a_{4}}\left(\alpha_{1}^{2}, \alpha_{2}^{2}, \alpha_{3}^{1}, \alpha_{4}^{2}\right)+\Pi_{a_{1} a_{2} a_{3} a_{4}}\left(\alpha_{1}^{2}, \alpha_{2}^{2}, \alpha_{3}^{2}, \alpha_{4}^{1}\right)+\Pi_{a_{1} a_{2} a_{3} a_{4}}\left(\alpha_{1}^{2}, \alpha_{2}^{2}, \alpha_{3}^{2}, \alpha_{4}^{2}\right),
\end{aligned}
$$

where $\alpha_{j}^{i}$ with $i=1,2$ and $j=1,2,3,4$ are two experimental settings of the coherent displacements for mode $a_{1}, a_{2}, a_{3}$ and $a_{4}$ respectively. By setting the experimental values of the coherent displacement as $\alpha_{1}^{1}=\alpha_{2}^{1}=$ $\alpha_{3}^{1}=\alpha_{4}^{1}=-0.104749, \alpha_{1}^{2}=\alpha_{2}^{2}=\alpha_{3}^{2}=\alpha_{4}^{2}=0.294117$, the maximum value of $\mathcal{B}_{4 \text { qubits }}$ is calculated numerically to be 5.14529 . Since local realistic theories require $\mathcal{B}_{4 q u b i t s} \leq 4$, the violation of local realism is simply shown from ŻB inequality.

It should be mentioned that other factors should be taken into account, such as detector inefficiencies, in practice. If one considers the inefficiency of detector, one has to modify the correlation slightly to account for the imperfections, which are characterized by the quantum efficiency of detectors $\eta(0 \leq \eta \leq 1)$. For ideal detectors, $\eta=1$ and the correlation is perfect. For non-ideal detectors, the correlation is modified by $\Pi_{a_{1} \ldots a_{N}}^{\prime}\left(\alpha_{1} \ldots \alpha_{N}\right)$. If we assume that all the photon detectors have the same efficiencies $\eta$,

$$
\begin{aligned}
& \Pi_{a_{1} a_{2} \ldots a_{N}}^{\prime}\left(\alpha_{1}, \alpha_{2}, \ldots, \alpha_{N}\right)= \\
& \left\langle\psi(N)\left|\hat{\Pi}_{a_{1}}^{\prime}\left(\alpha_{1}\right) \otimes \hat{\Pi}_{a_{2}}^{\prime}\left(\alpha_{2}\right) \otimes \ldots \otimes \hat{\Pi}_{a_{N}}^{\prime}\left(\alpha_{N}\right)\right| \psi(N)\right\rangle,
\end{aligned}
$$

where $\hat{\Pi}_{a_{i}}^{\prime}\left(\alpha_{i}\right)$ is an operator defined as

$$
\begin{aligned}
\hat{\Pi}_{a_{i}}^{\prime}\left(\alpha_{i}\right) & =\hat{D}_{a_{i}}\left(\alpha_{i}\right)(-1)^{\eta \hat{n}_{a_{i}}} \hat{D}_{a_{i}}^{\dagger}\left(\alpha_{i}\right) \\
& =\hat{D}_{a_{i}}\left(\alpha_{i}\right)(1-2 \eta)^{\hat{n}_{a_{i}}} \hat{D}_{a_{i}}^{\dagger}\left(\alpha_{i}\right) .
\end{aligned}
$$

A straightforward calculation yields the modified correlation function for the state $\psi(N)\rangle$

$$
\begin{aligned}
& \Pi_{a_{1} a_{2} \ldots a_{N}}^{\prime}\left(\alpha_{1}, \alpha_{2}, \ldots, \alpha_{N}\right)= \\
& \frac{1}{N}\left\{(-2 \eta)^{2}\left|\sum_{i=1}^{N} \alpha_{i}\right|^{2}+N(1-2 \eta)\right\} e^{-2 \eta \sum_{i=1}^{N}\left|\alpha_{i}\right|^{2}}(17)
\end{aligned}
$$

The detector efficiency directly affects the experimental results. As an example, we consider the 3-qubit experiment. Using the modified correlation functions

$$
\begin{aligned}
& \Pi_{a_{1} a_{2} a_{3}}^{\prime}\left(\alpha_{1}, \alpha_{2}, \alpha_{3}\right)= \\
& \frac{1}{3}\left\{(-2 \eta)^{2}\left|\sum_{i=1}^{3} \alpha_{i}\right|^{2}+3(1-2 \eta)\right\} e^{-2 \eta \sum_{i=1}^{3}\left|\alpha_{i}\right|^{2}} \\
& \Pi_{a_{i} a_{j}}^{\prime}\left(\alpha_{i}, \alpha_{j}\right)= \\
& \frac{1}{3}\left\{(-2 \eta)^{2}\left(\left|\alpha_{i}+\alpha_{j}\right|^{2}\right)+3-4 \eta\right\} e^{-2 \eta\left(\left|\alpha_{i}\right|^{2}+\left|\alpha_{j}\right|^{2}\right)} \\
& \Pi_{a_{i}}^{\prime}\left(\alpha_{i}\right)= \\
& \frac{1}{3}\left\{(-2 \eta)^{2}\left(\left|\alpha_{i}\right|^{2}\right)+3-2 \eta\right\} e^{-2 \eta\left(\left|\alpha_{i}\right|^{2}\right)}
\end{aligned}
$$

where $i, j=1,2,3$, a Bell quantity $\mathcal{B}_{3 \text { qubits }}^{\eta}$ is constructed from the Bell inequality given in Ref. [15],

$$
\begin{aligned}
\mathcal{B}_{3 \mathrm{qubits}}^{\eta} & =-\Pi_{a_{1} a_{2} a_{3}}^{\prime}\left(\alpha_{1}^{1}, \alpha_{2}^{1}, \alpha_{3}^{1}\right)+\Pi_{a_{1} a_{2} a_{3}}^{\prime}\left(\alpha_{1}^{1}, \alpha_{2}^{1}, \alpha_{3}^{2}\right) \\
& +\Pi_{a_{1} a_{2} a_{3}}^{\prime}\left(\alpha_{1}^{1}, \alpha_{2}^{2}, \alpha_{3}^{1}\right)+\Pi_{a_{1} a_{2} a_{3}}^{\prime}\left(\alpha_{1}^{2}, \alpha_{2}^{1}, \alpha_{3}^{1}\right) \\
& -\Pi_{a_{1} a_{2} a_{3}}^{\prime}\left(\alpha_{1}^{2}, \alpha_{2}^{2}, \alpha_{3}^{2}\right)-\Pi_{a_{1} a_{2}}^{\prime}\left(\alpha_{1}^{1}, \alpha_{2}^{2}\right) \\
& -\Pi_{a_{1} a_{2}}^{\prime}\left(\alpha_{1}^{2}, \alpha_{2}^{1}\right)-\Pi_{a_{1} a_{2}}^{\prime}\left(\alpha_{1}^{2}, \alpha_{2}^{2}\right) \\
& -\Pi_{a_{1} a_{3}}^{\prime}\left(\alpha_{1}^{1}, \alpha_{3}^{2}\right)-\Pi_{a_{1} a_{3}}^{\prime}\left(\alpha_{1}^{2}, \alpha_{3}^{1}\right) \\
& -\Pi_{a_{1} a_{3}}^{\prime}\left(\alpha_{1}^{2}, \alpha_{3}^{2}\right)-\Pi_{a_{2} a_{3}}^{\prime}\left(\alpha_{2}^{1}, \alpha_{3}^{2}\right) \\
& -\Pi_{a_{2} a_{3}}^{\prime}\left(\alpha_{2}^{2}, \alpha_{3}^{1}\right)-\Pi_{a_{2} a_{3}}^{\prime}\left(\alpha_{2}^{2}, \alpha_{3}^{2}\right) \\
& +\Pi_{a_{1}}^{\prime}\left(\alpha_{1}^{1}\right)+\Pi_{a_{2}}^{\prime}\left(\alpha_{2}^{1}\right)+\Pi_{a_{3}}^{\prime}\left(\alpha_{3}^{1}\right) .
\end{aligned}
$$

Since, for a local realistic description, $\mathcal{B}_{3 \text { qubits }}^{\eta} \leq 3$ and for quantum nonlocality, the Bell quantity $\mathcal{B}_{3 \text { qubits }}^{\eta}$ has a maximum value of 3.1605 , which is greater than 3 when $\eta>0.9804$. Thus, the nonlocality of a three-qubit system exhibits in the proposed experiment if $\eta>0.9804$. Quantum nonlocality of $\mathrm{N}$ qubits ( $\mathrm{N}$ is an arbitrary number) 
can be tested in the proposed experiment in a similar way.

In the article, we propose an experimental setup for testing quantum nonlocality of $N$ qubits by using $N$ qubit Bell inequalities. The correlation function measured in the scheme is described by the Wigner function.
We also briefly consider the case in which the detectors are inefficient and provide a threshold value above which nonlocality observation can be obtained.

This work is supported by NUS academic research Grant No. WBS: R-144-000-123-112.
[1] K. Banaszek and K. Wódkiewicz, Phys. Rev. Lett. 82, 2009 (1999).

[2] A. Aspect, P. Grangier and G. Roger, Phys. Rev. Lett. 47, 460 (1981); ibid 49, 91 (1982); A. Aspect, J. Dalibard and G. Roger, ibid 49, 1804 (1982).

[3] G. Weihs, T. Jennewein, C. Simon, H. Weinfurter, and A. Zeilinger, Phys. Rev. Lett. 81, 5039 (1998); A. Aspect, Nature 398, 189 (1999).

[4] J. W. Pan, D. Bouwmeester, M. Daniell, H. Weinfurter, and A. Zeilinger, Nature 403, 515 (2000).

[5] Z. Zhao, T. Yang, Y. A. Chen, A. N. Zhang, M. Żukowski, and J. W. Pan, Phys. Rev. Lett. 91, 180401 (2003).

[6] J. F. Clauser, M. A. Horne, A. Shimony, and R. A. Holt, Phys. Rev. Lett. 23, 880 (1969).

[7] N. D. Mermin, Phys. Rev. Lett. 65, 1838 (1990).

[8] M. Ardehali, Phys. Rev. A 46, 5375 (1992).

[9] A. V. Belinskii and D. N. Klyshko, Phys. Usp. 36, 653
(1993).

[10] R. F. Werner and M. M. Wolf, Phys. Rev. A 64, 032112 (2001).

[11] M. Żukowski and Č. Brukner, Phys. Rev. Lett. 88, 210401 (2002).

[12] N. Gisin, Phys. Lett. A 154, 201 (1991); N. Gisin and A. Peres, Phys. Lett. A 162, 15 (1992).

[13] M. Żukowski, Č. Brukner, W. Laskowski, and M. Wiesniak, Phys. Rev. Lett. 88, 210402 (2002).

[14] J. L. Chen, C. F. Wu, L. C. Kwek and C. H. Oh, Phys. Rev. Lett. 93140407 (2004).

[15] J. L. Chen, C. F. Wu, L. C. Kwek and C. H. Oh, e-print: quant-ph/0507227

[16] S. L. Braunstein, A. Mann, and M. Revzen, Phys. Rev. Lett. 68, 3259 (1992). 